\title{
Cluster synchronization modes in an ensemble of coupled chaotic oscillators
}

\author{
Belykh, Vladimir N.; Belykh, Igor V.; Mosekilde, Erik
}

Published in:

Physical Review E. Statistical, Nonlinear, and Soft Matter Physics

Link to article, DOI:

10.1103/PhysRevE.63.036216

Publication date:

2001

Document Version

Publisher's PDF, also known as Version of record

Link back to DTU Orbit

Citation (APA):

Belykh, V. N., Belykh, I. V., \& Mosekilde, E. (2001). Cluster synchronization modes in an ensemble of coupled chaotic oscillators. Physical Review E. Statistical, Nonlinear, and Soft Matter Physics, 63(3), 036216. https://doi.org/10.1103/PhysRevE.63.036216

\section{General rights}

Copyright and moral rights for the publications made accessible in the public portal are retained by the authors and/or other copyright owners and it is a condition of accessing publications that users recognise and abide by the legal requirements associated with these rights.

- Users may download and print one copy of any publication from the public portal for the purpose of private study or research.

- You may not further distribute the material or use it for any profit-making activity or commercial gain

- You may freely distribute the URL identifying the publication in the public portal 


\title{
Cluster synchronization modes in an ensemble of coupled chaotic oscillators
}

\author{
Vladimir N. Belykh, ${ }^{1}$ Igor V. Belykh, ${ }^{2}$ and Erik Mosekilde ${ }^{3}$ \\ ${ }^{1}$ Advanced School of Physics, Nizhny Novgorod University, 23, Gagarin Avenue, Nizhny Novgorod, 603600 Russia \\ ${ }^{2}$ Institute for Applied Mathematics and Cybernetics, 10, Ul'yanov Street, Nizhny Novgorod, 603005 Russia \\ ${ }^{3}$ Department of Physics, Technical University of Denmark, 2800 King's Lyngby, Denmark
}

(Received 13 June 2000; published 27 February 2001)

\begin{abstract}
Considering systems of diffusively coupled identical chaotic oscillators, an effective method to determine the possible states of cluster synchronization and ensure their stability is presented. The method, which may find applications in communication engineering and other fields of science and technology, is illustrated through concrete examples of coupled biological cell models.
\end{abstract}

DOI: 10.1103/PhysRevE.63.036216

PACS number(s): 05.45.Xt, 05.45.Jn, 87.18.Hf

Studying the emergence of coherent spatiotemporal patterns in large ensembles of coupled nonlinear systems is a fundamental problem in theoretical physics with application to many areas of science. The simplest mode of such spatiotemporal patterns in a discrete medium of coupled identical oscillators with chaotic dynamics is full synchronization [1-9]. Here all oscillators of the ensemble acquire identical chaotic behaviors even though their initial conditions may be different. Cluster synchronization is observed when the oscillators synchronize with one another in groups, but there is no synchronization among the groups [10-15]. Oscillators with identical temporal dynamics form one cluster.

An intriguing problem in the study of such spatiotemporal patterns is how to select a particular structure from the whole collection of possible modes and to provide for its stability. This is of interest, for instance, in connection with many applications in communication engineering [16].

We have recently presented a family of embedded synchronization manifolds defining the cluster synchronization in the wide class of locally coupled identical systems $[14,15]$. In this paper, we consider the phenomenon of clustering in an array of nonlocally coupled oscillators described by the following system:

$$
\dot{X}=F(X)+(\mathcal{E} G \otimes P) X
$$

where $X=\left(X_{1}, X_{2}, \ldots, X_{N}\right)^{T}$ is the set of dynamical variables of $N$ oscillators forming the array, $X_{i}$ is the $m$-dimensional vector of the $i$ th oscillator variables, and $F(X)=\left(F\left(X_{1}\right), F\left(X_{2}\right), \ldots, F\left(X_{N}\right)\right)^{T}$. Elements of the $m$ $\times m$ matrix $P$ that are equal to 1 determine by which variables the oscillators are coupled. The matrix $\mathcal{E}$ is an $N \times N$ matrix with elements $\varepsilon_{i j}$ that define the nonlocal type of coupling as well as the coupling strengths between the oscillators. $G$ is the $N \times N$ diffusion or nearest-neighbor coupling matrix

$$
G=\left(\begin{array}{ccccc}
-1 & 1 & 0 & \ldots & 0 \\
1 & -2 & 1 & \ldots & 0 \\
\vdots & \vdots & \vdots & \vdots & \vdots \\
0 & \ldots & 0 & 1 & -1
\end{array}\right), \quad P=\left(\begin{array}{ccc}
1 & 0 & 0 \\
0 & 0 & 0 \\
0 & 0 & 0
\end{array}\right)
$$

A peculiarity of this type of nonlocal coupling is that each element of the array in principle is coupled with each other through its local diffusive interaction with the nearest neighbors. Note, however, that the matrix $A \equiv \mathcal{E} G$ is an arbitrary $N \times N$ matrix, and that the system (1) may be considered as an ensemble of $N$ oscillators with an arbitrary global coupling having the property that the sum of all elements of each line of the matrix $A$ equals to zero. This implies the possibility of the full synchronization mode. In the case that the diagonal matrix $\mathcal{E}=\mathcal{E}_{e} \equiv \varepsilon I_{N}$, where $I_{N}$ is a unit $N \times N$ matrix, the system (1) becomes a system of diffusively (locally) coupled oscillators in a chain with zero-flux boundary conditions, and written in a form similar to Heagy et al. [5], Wu and Chua [6], or Pecora [9].

Our main objective is to show that in the case when the locally coupled systems with the matrix $\mathcal{E}_{e}$ do not exhibit stable cluster synchronization and only full (one-cluster) synchronization occurs, the family $\mathcal{E}$ of nonlocally coupled systems may exhibit the desired cluster synchronization patterns whereas the state of full synchronization is unstable.

We first present the conditions on the coupling matrix $\mathcal{E}$ for the globally coupled system (1) to have the same cluster synchronization manifolds as the locally coupled system (1) with $\mathcal{E}=\varepsilon I_{N}$ [14]. Denote $E_{n}=I_{n} \otimes I_{m}$ and $\bar{E}_{n}=\bar{I}_{n} \otimes I_{m}$, where $I_{n}$ and $I_{m}$ are unit matrices, and $\bar{I}_{n}$ is an $n \times n$ matrix whose nonzero elements are all equal to one and lie in the secondary diagonal. $C_{e}=\left(E_{n} \bar{E}_{n}\right)^{T}$ for an even number of oscillators $N=2 n$,

$$
C_{o}=\left(\begin{array}{cc}
E_{n-1} & 0 \\
0 & I_{m} \\
\bar{E}_{n-1} & 0
\end{array}\right)
$$

for odd $N=2 n-1, C_{a}=\left(E_{n}, \bar{E}_{n}, E_{n}, \ldots\right)^{T}$, where $E_{n}$ alternating with $\bar{E}_{n}$ is repeated $r$ times, for $N=r n$, and $U$ $=\left(U_{1}, U_{2}, \ldots, U_{n}\right)^{T}$. The following assertion then holds.

Proposition. Let the matrix $\mathcal{E} C_{s}$ be defined as the first $n$ lines of the matrix $\mathcal{E} C_{s}$ and let

$$
C_{s}\left(\underline{\mathcal{E} C_{s}}\right)=\mathcal{E} C_{s},
$$


where $s$ stands for $o, e$, and $a$. Then the system (1) with the global coupling matrix $\mathcal{E}$ has the following $n$-cluster synchronization manifolds: $M^{c}(2 n, n)=\left\{X=C_{e} U\right\}$ for even $N$ $=2 n, M^{c}(2 n-1, n)=\left\{X=C_{o} U\right\}$ for odd $N=2 n-1$, and $M^{a}(r n, n)=\left\{X=C_{a} U\right\}$ for $N=r n$.

Condition (2) implies that to preserve these manifolds in the case of global coupling, the matrix $\mathcal{E}$, which in the general case is filled out by nonzero elements, must be such that those lines of the matrix $\mathcal{E} C_{s}$ that belong to the same cluster are identical.

We shall illustrate the conditions of the proposition through an example with $N=5$ oscillators. In the case of local diffusive coupling, the system (1) (with the matrices $\mathcal{E}_{e}$ and $G$ ) has only two in-phase synchronization manifolds: the diagonal $M(5,1)=\left\{X_{1}=X_{2}=\cdots=X_{5}\right\}$ that corresponds to full synchronization of the oscillators (one-cluster synchronization) and the manifold $M^{c}(5,3)=\left\{X_{1}=X_{5}, X_{2}=X_{4}\right\}$ that determines the central symmetry of synchronized oscillators in pairs with respect to the middle (third) non-synchronized element, i.e., a three-cluster synchronization manifold.

In the case of global "diffusive" coupling, by virtue of the proposition, the cluster manifold $M^{c}(5,3)=\left\{X_{1}=X_{5}, X_{2}\right.$ $\left.=X_{4}\right\}$ does exist iff each sum of pairs of column elements symmetrical with respect to the middle one has similar pairs of the elements (sums of pairs) symmetrical with respect to the middle row.

As an illustration, we present the matrix $C_{o}$ that defines the cluster manifold $M^{c}(5,3)$ and a curious case of the matrix $\mathcal{E}$ :

$$
\mathcal{E}=\left(\begin{array}{lllll}
1 & 9 & 4 & 3 & 5 \\
9 & 6 & 5 & 2 & 0 \\
4 & 1 & 9 & 7 & 3 \\
1 & 7 & 5 & 1 & 8 \\
4 & 7 & 4 & 5 & 2
\end{array}\right), \quad C_{o}=\left(\begin{array}{lll}
1 & 0 & 0 \\
0 & 1 & 0 \\
0 & 0 & 1 \\
0 & 1 & 0 \\
1 & 0 & 0
\end{array}\right)
$$

At first sight, the matrix $\mathcal{E}$ seems to be disordered (arbitrary) and therefore to allow only the manifold $M(5,1)$ defining full synchronization. But in fact, the product $\mathcal{E} C_{o}$ satisfies condition (2), and the required manifold $M(5,3)$ exists and defines a cluster synchronization with three clusters (the elements of $\mathcal{E}=\left\{\varepsilon_{i j}\right\}_{i, j=1, \ldots, 5}$ satisfy the conditions $\varepsilon_{11}+\varepsilon_{15}$ $=\varepsilon_{51}+\varepsilon_{55}, \varepsilon_{21}+\varepsilon_{25}=\varepsilon_{41}+\varepsilon_{45}$ and so forth).

Numerous examples of locally coupled identical continuous time oscillators in a chain show that usually cluster synchronization regimes cannot be achieved via the change of a single coupling strength parameter, and only a few examples of locally coupled identical systems demonstrate such a cluster synchronization (for instance, $x$-coupled Rössler systems $[5,9,14])$. Now the problems arise of how to find the minimum number of nonzero elements of the matrix $\mathcal{E}$ providing conditions of both the existence and stability of cluster synchronization regimes defined by the synchronization manifolds from the local coupling case and how to realize the required stable cluster pattern from the whole collection of all possible modes.
As an effective method, we propose in this paper to consider the cross matrix $\mathcal{E}$ having only nonzero elements in the principal and secondary diagonals (all other elements are zeros). Such a matrix $\mathcal{E}$ defines a nontrivial nonlocal coupling between the oscillators. Note that the effectiveness of the cross matrix $\mathcal{E}$ with the two diagonals is obvious in the problem of the selection of the manifolds because we involve the oscillators forming the cluster in direct coupling interaction and thus we expect the emergence of all negative transversal Lyapunov exponents of the cluster manifold and of a positive transversal Lyapunov exponent of the diagonal manifold $M(N, 1)$. Hence, full synchronization does not occur.

Clustering of chaotic oscillators is considered to be particularly significant in the biological sciences where one often encounters coupled cells or functional units that each display a complicated nonlinear behavior [10]. As an example, we consider an array of coupled Sherman models for pancreatic $\beta$ cells [17]. The coupled Sherman models with scalar diffusive coupling and zero-flux boundary conditions are described by the system (1) with the matrices $G$ and $\mathcal{E}_{e}$ and the following individual oscillator equations:

$$
\begin{gathered}
\tau_{1} \frac{d V}{d t}=-I_{\mathrm{Ca}}(V)-I_{K}(V, n)-g_{S} S\left(V-V_{K}\right), \\
\tau_{2} \frac{d n}{d t}=\sigma\left[n_{\infty}(V)-n\right], \\
\tau_{S} \frac{d S}{d t}=S_{\infty}(V)-S,
\end{gathered}
$$

where

$$
\begin{gathered}
I_{\mathrm{Ca}}(V)=g_{\mathrm{Ca}} m_{\infty}(V)\left(V-V_{\mathrm{Ca}}\right), \\
I_{K}(V, n)=g_{K} n\left(V-V_{K}\right),
\end{gathered}
$$

and

$$
\omega_{\infty}(V)=\left[1+\exp \left\{\frac{V_{\omega}-V}{\theta_{\omega}}\right\}\right]^{-1} \text { for } \omega=m, n, \quad \text { and } S .
$$

Here, $V$ represents the membrane potential, $n$ is the opening probability of the potassium channels, and $S$ accounts for the presence of a slow dynamics in the system. $g_{\mathrm{Ca}}=3.6, V_{\mathrm{Ca}}$ $=25, V_{m}=-20, \theta_{m}=12, g_{\mathrm{K}}=10, V_{\mathrm{K}}=-75, g_{S}=4, \sigma$ $=1, V_{n}=-16, \theta_{n}=5.6, V_{S}=-35.245, \theta_{S}=10$, and $\tau_{1}$ $=0.004, \tau_{2}=0.0045, \tau_{S}=10$ are model parameters. $\tau_{1,2} / \tau_{S}$ defines the ratio of the fast ( $V$ and $n$ ) and the slow $(S)$ time scales. The individual Sherman model may display chaotic dynamics in a wide region of the parameters [17,18].

With the assumed coupling configuration the system (1) with the individual system (3) represents a chain of locally coupled pancreatic $\beta$ cells (resistive electrical coupling via the variables $V_{i}$ ). Similar to most other diffusively coupled systems, the coupled Sherman models do not exhibit the phenomenon of cluster synchronization when changing the coupling strength, and only full synchronization takes place. 

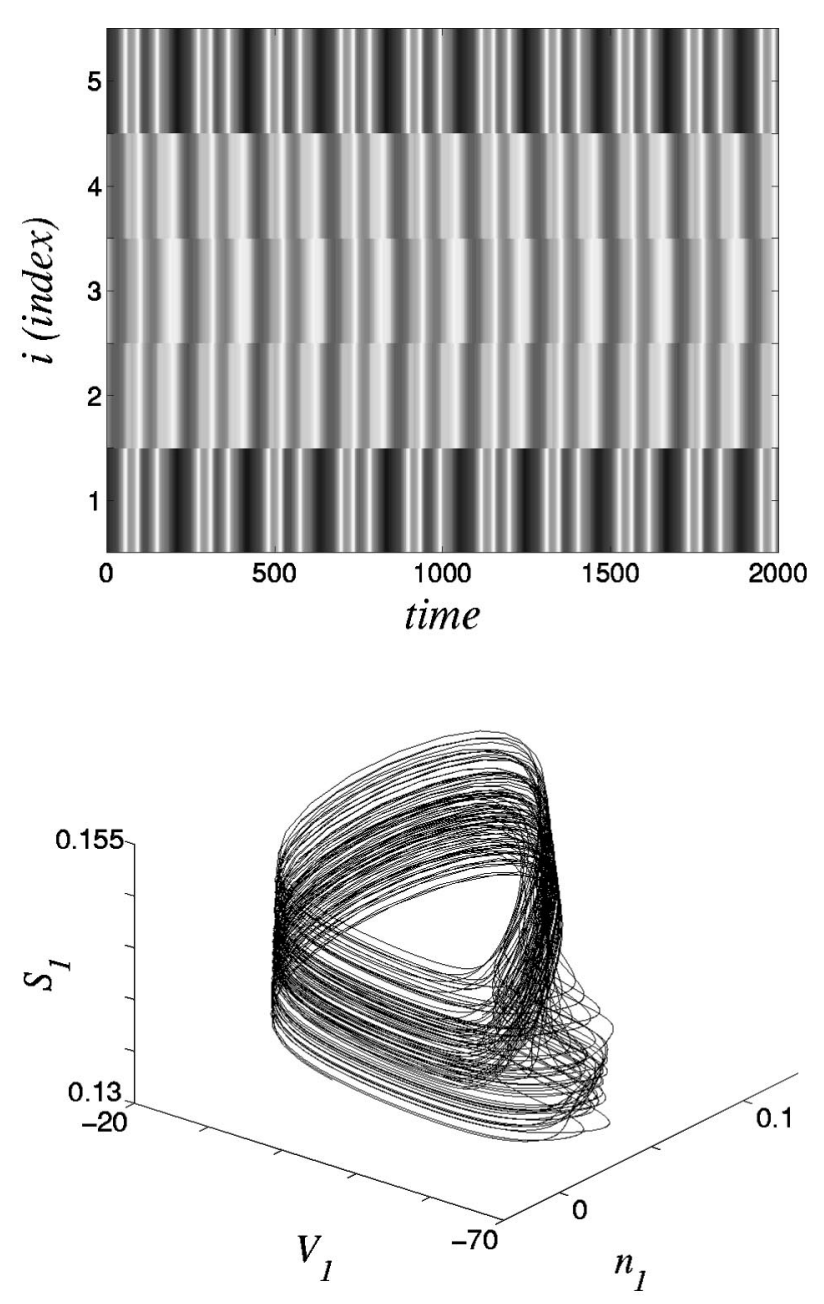

FIG. 1. Established cluster synchronization of five nonlocally coupled Sherman models. The coupling parameter $\varepsilon=4$. Different shades of gray represent different ranges of amplitudes of $V_{i}(t)$ (top). Chaotic attractor, in the phase space $\left(V_{1}, n_{1}, S_{1}\right)$, defining the temporal behavior of the first (and the fifth) cell in the regime of chaotic bursting behavior with cluster synchronization (bottom).

First, we consider five coupled Sherman models in the form (1)-(3). To select the cluster synchronization with three clusters defined by the existence of the manifold $M^{c}(5,3)$ $=\left\{X_{1}=X_{5}, X_{2}=X_{4}\right\}$, where $X_{i}=\left(V_{i}, n_{i}, S_{i}\right), i=\overline{1,5}$, we consider the cross matrix $\mathcal{E}$ that has its only nonzero elements lying in the two diagonals: $\varepsilon_{22}=\varepsilon_{33}=\varepsilon_{44}=\varepsilon$ and $\varepsilon_{15}=\varepsilon_{51}=-\varepsilon$. The system (1)-(3) with this coupling matrix $\mathcal{E}$ may be rewritten in the following simple form:

$$
\begin{gathered}
\dot{X}_{1}=F\left(X_{1}\right)-\varepsilon P\left(X_{4}-X_{5}\right), \\
\dot{X}_{2}=F\left(X_{2}\right)+\varepsilon P\left(X_{3}-2 X_{2}+X_{1}\right), \\
\dot{X}_{3}=F\left(X_{3}\right)+\varepsilon P\left(X_{4}-2 X_{3}+X_{2}\right), \\
\dot{X}_{4}=F\left(X_{4}\right)+\varepsilon P\left(X_{5}-2 X_{4}+X_{3}\right), \\
\dot{X}_{5}=F\left(X_{5}\right)-\varepsilon P\left(X_{2}-X_{1}\right) .
\end{gathered}
$$
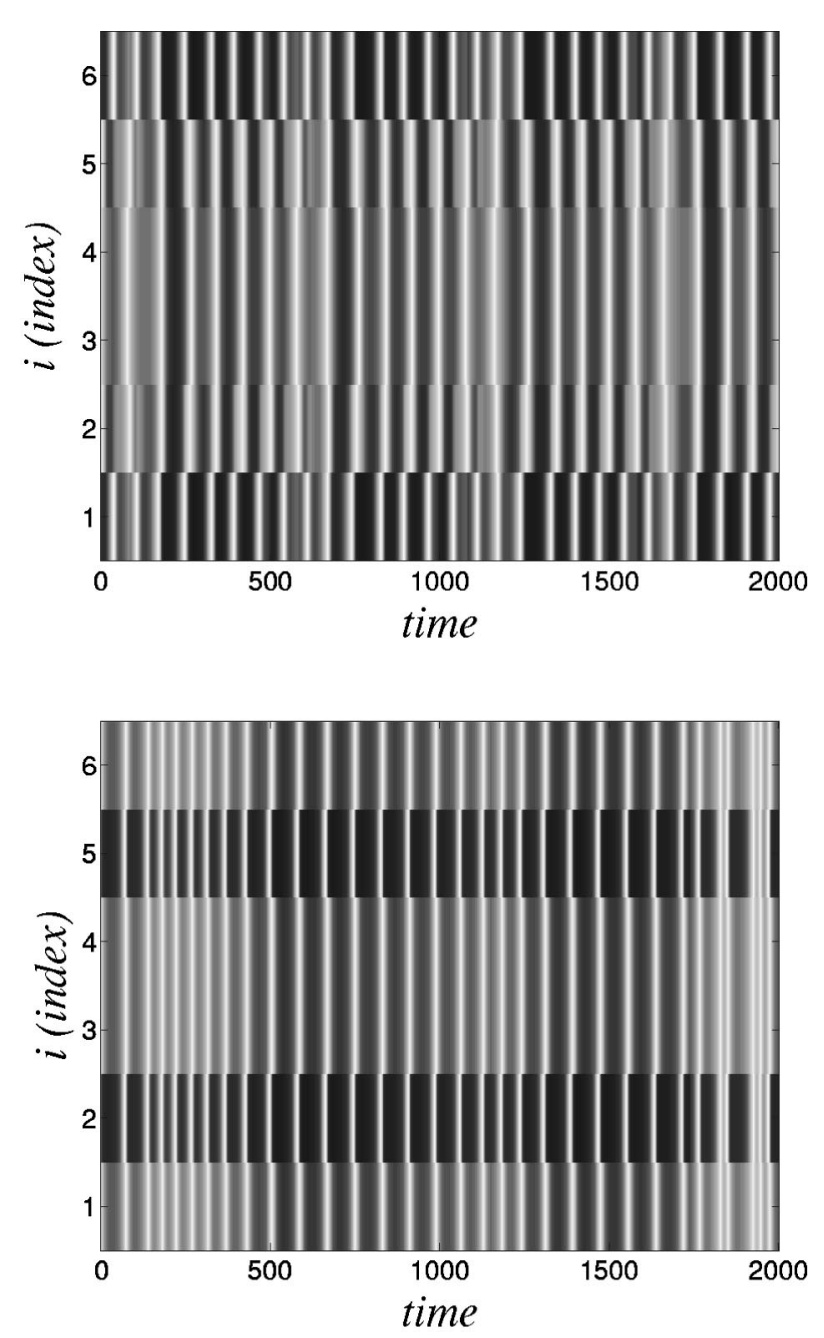

FIG. 2. Symmetrical cluster synchronization of six coupled Sherman models for different nonlocal coupling configurations. Three-cluster pattern defined by the manifold $M^{c}(6,3)$ (top). Coupling strength $\varepsilon=4$. Two-cluster pattern defined by the manifold $M^{c}(6,2)$ (bottom). The coupling parameters are $\varepsilon=6, \varepsilon_{1}=4$, and $\varepsilon_{2}=0.5$.

Numerical simulations confirm our assumptions about the transversal stability of the diagonal synchronization manifold, and the stable mode of cluster chaotic synchronization with three clusters is observed in the system over a fairly wide range of the coupling parameter $\varepsilon$ (see Fig. 1).

Consider now an array of six coupled Sherman models. For $N=6$, the system of locally coupled Sherman models [as well as the general system (1) with the matrix $\mathcal{E}_{e}$ ] aside from the diagonal $M(6,1)$, has the invariant manifold $M^{c}(6,3)$ $=\left\{X_{1}=X_{6}, X_{2}=X_{5}, X_{3}=X_{4}\right\}$, which defines the same central symmetry of synchronized oscillators with respect to the middle of the array as in the previous example. But in contrast to the case $N=5$, two additional invariant manifolds $M^{c}(6,2)=\left\{X_{1}=X_{3}=X_{4}=X_{6}, X_{2}=X_{5}\right\}$ and $M^{a}(6,2)=\left\{X_{1}\right.$ $\left.=X_{4}=X_{5}, X_{2}=X_{3}=X_{6}\right\}$ exist. Therefore, arrays of the form (1) that are composed from five and from six diffusively coupled elements may exhibit completely different regimes of cluster synchronization.

Our purpose is to select and provide the stability of all 


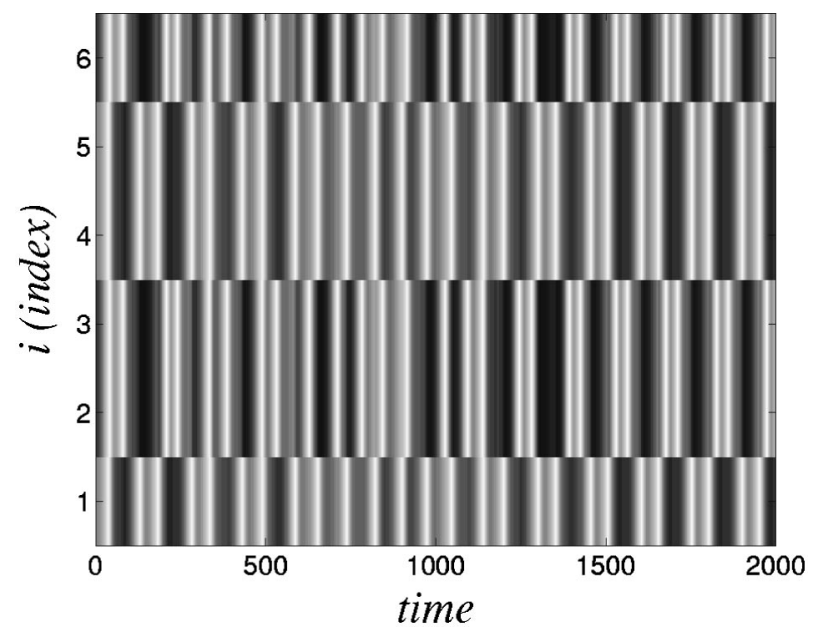

FIG. 3. Nonsymmetrical two-cluster synchronization pattern defined by the manifold $M^{a}(6,2)$. The coupling parameters are $\varepsilon$ $=1.3, \varepsilon_{1}=2$, and $\varepsilon_{2}=3.4$.

cluster synchronization manifolds $M^{c}(6,3), M^{c}(6,2)$, and $M^{a}(6,2)$ by introducing the nonlocal coupling defined by the cross matrix $\mathcal{E}$.

First we select the cluster dynamical regime defined by the manifold $M^{c}(6,3)$. Similar to the case $N=5$ we introduce the nonlocal coupling between the oscillators using the 6 $\times 6$ cross matrix $\mathcal{E}$ with the only nonzero elements lying in the two diagonals $\varepsilon_{22}=\varepsilon_{33}=\varepsilon_{44}=\varepsilon_{55}=\varepsilon$ and $\varepsilon_{16}=\varepsilon_{61}=$ $-\varepsilon$. Note that due to the proposition, the manifold $M^{c}(6,3)$ and its submanifold $M^{c}(6,2)$ of the system with local coupling are preserved for such a nonlocal coupling but the manifold $M^{a}(6,2)$, defining nonsymmetrical clusters of synchronized oscillators, no longer exists. It is shown in Fig. 2 (top) that the required cluster synchronization regime defined by the manifold $M^{c}(6,3)$ is indeed realized in the six coupled Sherman models in some region of the coupling parameter. The cells of the array are synchronized in three pairs with respect to the middle of the array.

We study now the selection of the stable cluster regime that is determined by the manifold $M^{c}(6,2)$. For this pur- pose, we change the coupling configuration in the cross matrix $\mathcal{E}$ such that the elements are $\varepsilon_{11}=\varepsilon_{33}=\varepsilon_{44}=\varepsilon_{66}=\varepsilon$, $\varepsilon_{25}=\varepsilon_{52}=-\varepsilon_{1}$, and $\varepsilon_{22}=\varepsilon_{55}=\varepsilon_{2}$. The other elements of the matrix equal to zero. The required synchronization with two clusters of synchronized cells is observed in the system and remains stable in a fairly large region of coupling parameters $\left(\varepsilon, \varepsilon_{1}, \varepsilon_{2}\right)$ [see Fig. 2 (bottom)].

To complete the selection of all possible cluster synchronization regimes for the case $N=6$, we need to provide the existence and stability of the manifold $M^{a}(6,2)$ that is related to another alternating symmetry and defines the following clustering: the first, the fourth, and the fifth $\beta$ cells are synchronized and define one cluster and the others are involved in the second cluster. For this we consider the nonlocal coupling matrix $\mathcal{E}$ with the principal diagonal elements $\varepsilon_{11}=\varepsilon_{22}=\varepsilon_{33}=\varepsilon_{44}=\varepsilon_{55}=\varepsilon_{66}=\varepsilon$, and the only nonzero elements far from the principal diagonal $\varepsilon_{51}=\varepsilon_{41}=\varepsilon_{54}=-\varepsilon_{1}$, and $\varepsilon_{26}=\varepsilon_{32}=\varepsilon_{63}=-\varepsilon_{2}$. Note that after this change of coupling configuration, aside from the diagonal $M(6,1)$, there exists only one nonsymmetrical cluster manifold $M^{a}(6,2)$ and the symmetrical manifolds $M^{c}(6,3)$ and $M^{c}(6,2)$ are no longer preserved from the local coupling case. Thus we provide the selection of the cluster synchronization regime from the whole set of the possible cluster synchronization regimes. This coupling configuration does realize the stability of this cluster dynamical regime in some region of coupling parameters (see Fig. 3).

In summary, we have selected the structure of global coupling providing the persistence of all cluster manifolds existing in locally diffusively coupled systems. We have presented an effective method to provide the stability of cluster synchronization modes, which are unstable in the case of locally coupled systems, by introducing an additional nonlocal coupling, and we have shown the effectiveness of the method through concrete examples of coupled systems.

This work was supported in part by RFFI (Grant No. 9901-01126) and by grant "Universities of Russia', (No. 1905). I.V.B. and V.N.B. acknowledge support from the Danish Research Academy.
[1] H. Fujisaka and T. Yamada, Prog. Theor. Phys. 69, 32 (1983); 72, 885 (1984).

[2] V.S. Afraimovich, N.N. Verichev, and M.I. Rabinovich, Izv. Vuzov. Radiofiz. 29, 795 (1986).

[3] L.M. Pecora and T.L. Carroll, Phys. Rev. Lett. 64, 821 (1990).

[4] J.F. Heagy, T.L. Carroll, and L.M. Pecora, Phys. Rev. E 50, 1874 (1994).

[5] J.F. Heagy, L.M. Pecora, and T.L. Carroll, Phys. Rev. Lett. 74, 4185 (1994).

[6] C.W. Wu and L.O. Chua, IEEE Trans. Circuits Syst., I: Fundam. Theory Appl. 42, 494 (1995).

[7] M. Gade, Phys. Rev. E 5, 64 (1996).

[8] L.M. Pecora and T.L. Carroll, Phys. Rev. Lett. 80, 2109 (1998).

[9] L.M. Pecora, Phys. Rev. E 58, 347 (1998).
[10] K. Kaneko, Physica D 41, 137 (1990); 54, 5 (1991); 55, 368 (1992); 75, 55 (1994).

[11] K. Otsuka and Y. Sato, Phys. Rev. A 54, 4464 (1996).

[12] D.H. Zanette and A.S. Mikhailov, Phys. Rev. E 57, 276 (1998).

[13] M.I. Rabinovich, J.J. Torres, P. Varona, R. Huerta, and P. Weidman, Phys. Rev. E 60, R1130 (1999).

[14] V.N. Belykh, I.V. Belykh, and M. Hasler, Phys. Rev. E (to be published).

[15] V.N. Belykh, I.V. Belykh, N.L. Komrakov, and E. Mosekilde, Discrete Dynamics (Gordon and Breach, New York, 2000), Vol. 4, p. 245.

[16] N.F. Rulkov, Chaos 6, 262 (1996).

[17] A. Sherman, J. Rinzel, and J. Keizer, Biophys. J. 54, 411 (1988).

[18] A. Sherman, Bull. Math. Biol. 56, 811 (1994). 